\title{
RDUS
}

Revue de DROIT

UNIVERSITÉ DE SHERBROOKE

Titre : $\quad$ LA PROPRIÉTÉ INDIVISE DU CODE CIVIL DU QUÉBEC : QUELQUES CONSIDÉRATIONS FISCALES

Auteur(s): $\quad$ Luce SAMOISETTE

Revue : $\quad$ RDUS, 1993-1994, volume 24, numéro 1

Pages: $\quad 185-197$

ISSN : $\quad 0317-9656$

Éditeur: $\quad$ Université de Sherbrooke. Faculté de droit.

URI : $\quad$ http://hdl.handle.net/11143/13366

DOI : $\quad$ https://doi.org/10.17118/11143/13366 
Page vide laissée intentionnellement. 


\title{
CHRONIQUE : NOUVEAU CODE CIVIL DU QUÉBEC
}

\section{LA PROPRIÉTÉ INDIVISE DU CODE CIVIL DU QUÉBEC : QUELQUES CONSIDÉRATIONS FISCALES}

\author{
par Luce SAMOISETTE*
}

En 1991, le législateur fédéral a modifié la Loi de l'impôt sur le revenu en y ajoutant des paragraphes spécifiques relatifs aux conséquences fiscales qu'entraînent le partage d'un bien détenu en indivision. Ces règles ne concernent que l'impôt sur le revenu et ne peuvent donc pas être appliquées à d'autres législations de nature fiscale, telles les taxes à la consommation. À défaut de règles spécifiques, les conséquences fiscales d'un partage en vertu de ces lois sont déterminées par le droit interne de chaque province; ce qui ne concorde pas nécessairement avec les règles retenues par le législateur fédéral aux fins de l'impôt sur le revenu. Quelles sont ces conséquences fiscales et peuvent-elles influencer l'issue d'un partage? Afin de répondre à ces questions, l'auteure explicite d'abord les règles contenues dans la Loi de l'impôt sur le revenu puis examine les conséquences fiscales d'un partage soumis à la Loi sur la taxe d'accise et à la Loi concernant les droits sur les mutations immobilières.

In 1991, Parliament amended the Income Tax Act by adding specific rules pertaining to the partition of undivided property. Since these rules apply only to the Income Tax Act, the fiscal consequences of a partition of undivided property under other tax legislation, such as sales taxes, are determined by provincial laws which do not necessarily coincide with the rules enacted for income tax purposes. What are the tax consequences of a partition of undivided property under the Income Tax Act and other tax laws? Could these consequences influence the outcome of a partition? In order to answer these questions, the writer explains the rules contained in the Income Tax Act. She also examines the fiscal consequences of a partition in light of the Excise Tax Act and the Act Respecting Duties on Transfers of Immoveables.

*. $\quad$ Notaire et professeure à la Faculté de droit de l'Université de Sherbrooke. 


\section{SOMMAIRE}

Introduction

I.- Loi de l'impôt sur le revenu . . . . . . . . . . . . . . . 189

A) Situation fiscale antérieure au 13 juillet $1990 \ldots \ldots \ldots 189$

B) Situation depuis le 13 juillet $1990 \ldots \ldots \ldots \ldots \ldots \ldots . \ldots \ldots$

C) Situation depuis le 1er janvier $1994 \ldots \ldots \ldots \ldots \ldots . \ldots 193$

II.- $\quad$ Autres lois de nature fiscale . . . . . . . . . . . . . . . 193

A) Loi concernant les droits sur les mutations immobilières

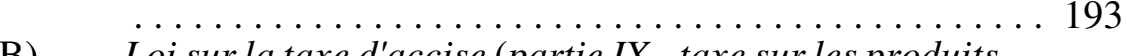

B) Loi sur la taxe d'accise (partie IX - taxe sur les produits

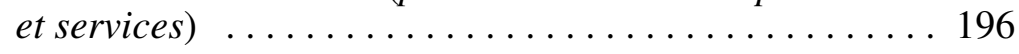

III.- Certaines dispositions du régime légal d'indivision à éviter . . 197

A) Le droit de retrait de l'article 1022 C.c.Q. . . . . . . . 197

B) La cessation de l'indivision (partage, art. 1033 C.c.Q.) . 198

Conclusion ............................. 198 


\section{Introduction}

Avant le 1er janvier 1994, la copropriété par indivision n'avait pas, au plan civil, de cadre légal organisé ${ }^{1}$. En réalité, le législateur considérait la copropriété par indivision comme un droit de propriété affaibli car partagé; en conséquence, il s'était contenté d'édicter une règle d'ordre public ${ }^{2}$ permettant aux parties d'y mettre fin facilement ${ }^{3}$. La copropriété par indivision étant une situation temporaire, tout indivisaire devait en effet avoir la certitude de pouvoir mettre fin à cet état dès qu'il le jugeait préférable à son entière discrétion. Une auteure présente ainsi l'état du droit antérieur :

«On peut aussi expliquer ces limitations [à l'indivision] lorsqu'on constate que les codificateurs n'ont prévu cette situation que dans un cadre restreint, principalement dans le cadre des successions. Il est alors compréhensible qu'ils n'aient pas voulu adopter une attitude trop interventionniste là où les membres d'une même famille auraient pu en venir à une entente entre eux pour conserver en commun des biens familiaux pour le temps qui leur convenait. En ce sens, les codificateurs ont fait confiance à la capacité des indivisaires de réglementer entre eux l'indivision lorsqu'il y avait consensus pour la prolonger. Les règles qu'ils ont prévues visent donc principalement à protéger la volonté de ceux des indivisaires qui ne voudraient pas prolonger une situation qu'ils n'ont pas recherchée. L'indivision est alors perçue comme une situation transitoire. ${ }^{4} »$

Cette conception de l'indivision ne reflétait cependant plus la réalité. Pour des raisons économiques ${ }^{5}$, l'indivision était devenue un mode de détention des biens de plus en plus populaire. Le législateur se devait donc de reconnaître l'indivision volontaire comme un mode de détention de biens pouvant s'étendre sur une longue période ${ }^{6}$. Une intervention de sa part était souhaitée et

1. $\quad$ Serge BINETTE, « De la propriété indivise et divise suivant le nouveau Code civil du Québec» dans La Réforme du Code civil, vol.1, Québec, Les Presses de l'Université Laval, 1993, p. 592.

2. Outre le cas des successions, art. 746 et ss. Code civil du Bas Canada (ci-après appelé C.c.B.C.).

3. L'article 689 C.c.B.C. stipulait que : «Nul ne peut être contraint à demeurer dans l'indivision; le partage peut toujours être provoqué nonobstant prohibition et convention contraires.»

4. Marie DESCHAMPS, «Vers une approche renouvelée de l'indivision», (1983-84) 29 R.D. Mc Gill 215, p. 234.

5. La copropriété indivise permet de refléter la réalité économique vécue par les indivisaires qui, sans l'existence de ce mode de propriété, n'auraient pas eu les moyens d'acquérir une propriété.

6. Â titre d'exemples, les conjoints québécois, mariés ou non, acquièrent de plus en plus fréquemment la résidence familiale et les meubles qui la garnissent en indivision. Il peut en être de même pour l'acquisition d'immeubles à revenus ou d'entreprises. À ce sujet, Marie Deschamps écrivait en 1984 :«Les juristes québécois semblent avoir pris conscience de 
souhaitable ${ }^{7}$ afin d'assurer la protection des indivisaires et des tiers. À ce sujet, le professeur Binette écrivait: :

«L'expérience prouve qu'il y a des indivisions qui durent à partir d'une décision expresse et éclairée, soit d'un consentement tacite. Mais vu l'inorganisation en ce domaine, les indivisaires se trouvent toujours devant des obstacles majeurs. ${ }^{8} \gg$

Au plan fiscal, ce n'est qu'en $1990^{9}$ que le législateur fédéral a édicté des règles spécifiques relatives aux partages. Ce faisant, il a volontairement choisi d'ignorer le droit interne des provinces pour établir sa propre vision d'un partage. Les mêmes règles ont été retenues par le législateur provincial ${ }^{10}$. Cependant, ces nouvelles règles ne concernent que l'impôt sur le revenu; elles ne s'appliquent donc pas aux autres législations fiscales telles les taxes à la consommation, les mutations immobilières, etc. À défaut de règles spécifiques, l'impact fiscal d'un partage en vertu de ces lois sera déterminé par les nouvelles dispositions civiles entrées en vigueur le 1er janvier 1994; ces dispositions, nous le verrons, ne concordent pas toujours avec les règles établies dans la Loi de l'impôt sur le revenu.

Afin de bien comprendre cette problématique, nous diviserons notre texte en trois parties. Dans la première partie, nous expliciterons les règles fiscales applicables lors d'un partage, tant celles applicables avant le 13 juillet 1990 qu'après. Ensuite, nous examinerons l'impact de deux lois de nature fiscale sur les partages, la Loi concernant les droits sur les mutations immobilières ${ }^{11}$ et la Loi sur la taxe d'accise ${ }^{12}$. Enfin, nous présenterons les conséquences fiscales entraînées par la mise en vigueur des dispositions relatives à l'indivision du Code civil du Québecc ${ }^{13}$. Il nous sera possible, au terme de notre étude, de démontrer l'impact important de la fiscalité en ce domaine, et l'influence que

l'évolution des mentalités et des changements dans la situation économique. Les dispositions du Projet de Code civil et du Projet 58 en sont la marque.» supra, note 4, p. 256.

7. Afin de pallier au manque de règles en cette matière, la Chambre des Notaires du Québec proposait, dans ses formulaires, des conventions d'indivision régissant les droits et les obligations des indivisaires durant et à la fin de l'indivision. Voir R.D. - BIENS Formulaire - Document 2.

8. Supra, note 1 .

9. Paragr. 248 (20) ajouté par L.C. 1991, chap. 49, art. 192 (18) applicable aux partages effectués après le 13 juillet 1990. Le paragraphe 248 (20) Loi de l'impôt sur le revenu, S.R.C. 1952, c. 148 modifiée par S.C. 1970-71-72 C- 63, (ci-après appelée L.I.R.) est reproduit en annexe.

10. Articles 2.1.1 et 2.1.2, Loi sur les Impôts, L.R.Q., c. I-3.

11. L.R.Q., c. D-15.1.

12. L.C. 1990, c. 45, telle qu'amendée, (Partie IX - TAXE SUR LES PRODUITS ET SERVICES), ci-après appelée L.T.A. ou T.P.S.

13. Arts 1012 à 1037 C.c.Q. 
celle-ci peut avoir dans certaines circonstances sur la rédaction des conventions d'indivision ${ }^{14}$.

\section{I.- $\quad$ Loi de l'impôt sur le revenu}

\section{A) Situation fiscale antérieure au 13 juillet $1990^{15}$}

Jusqu'en juillet 1990, la Loi de l'impôt sur le revenu ne contenait pas de règles spécifiques au partage d'un bien détenu en indivision. L'impact fiscal d'un partage entre indivisaires était donc dépendant des règles civiles qui le régissaient. Ainsi, si le droit d'une province considérait le partage comme translatif de propriété, il y avait disposition de biens ${ }^{16}$ aux fins de la Loi de l'impôt sur le revenu et les indivisaires devaient, le cas échéant, inclure le gain en capital imposable dans le calcul de leur revenu. À l'inverse, si le droit provincial décrètait qu'un partage était plutôt déclaratif du droit de propriété, il n'y avait aucun transfert au sens «civil» et, par conséquent, aucune disposition au plan fiscal. À cette époque, au Québec, un partage était déclaratif du droit de propriété. Suite au partage, chacun des indivisaires était donc considéré avoir toujours été propriétaire de la partie du bien reçu depuis la date d'acquisition ${ }^{17}$ de sa part indivise.

En pratique, cette situation pouvait créer des abus. Prenons un exemple : Louis et Philippe sont copropriétaires indivis, en parts égales, de deux biens. Le bien «A» a une valeur marchande de 40000 . , alors que le bien «B» a une valeur marchande de 60000 . Chacun des biens avait été acquis pour la somme de 20 000.\$. Louis et Philippe procèdent au partage des biens qu'ils détiennent en indivision en établissant que Philippe est maintenant seul propriétaire du bien «A» et que Louis est seul propriétaire du bien «B». Dans cet exemple, Louis aurait compensé Philippe en argent, soit par le paiement d'une soulte au montant de 10 000.\$. Un tel partage n'engendrait aucune conséquence fiscale pour l'un ou l'autre des indivisaires et ce, bien que Philippe ait reçu, en plus du bien «A», une somme de 10 000.\$ en paiement de sa part indivise. C'est pour pallier à de pareilles lacunes que le législateur a adopté des règles fiscales en la matière.

14. Nous ne discuterons pas de partage successoral ou de partage entre conjoints puisque d'autres dispositions particulières de la L.I.R. les régissent, 248 (22) et (23) L.I.R.

15. Notre étude traite uniquement de l'impact fiscal occasionné par le partage d'un bien en immobilisation au sens de l'article 54 L.I.R.

16. Art. 54 L.I.R.

17. Denys-Claude LAMONTAGNE, «Étude des principaux cas de transfert et d'exonération aux termes de la Loi autorisant les municipalités à percevoir un droit sur les mutations immobilières», (1988) 90 R. du N. 501, p. 506. Voir également supra, note 4, pp. 224 et 247. 


\section{B) Situation depuis le 13 juillet 1990}

En $1991^{18}$, le législateur fédéral a ajouté à la Loi de l'impôt sur le revenu les paragraphes qui élaborent les règles fiscales applicables lors d'un partage entre indivisaires. Ces dispositions uniformisent les conséquences d'un partage à travers tout le Canada et surtout éliminent certains abus. Depuis le 13 juillet 1990, le partage d'un bien détenu en indivision n'entraînera aucune conséquence fiscale si la valeur de la partie du bien reçue suite au partage est égale à la valeur de la part indivise dans ce même bien de l'indivisaire avant le partage. En conséquence, une diminution de valeur entraînera une disposition réputée et une augmentation de valeur, une acquisition réputée. Illustrons ces nouvelles règles à l'aide d'exemples.

EXEMPLE 1 : André et Bernard sont copropriétaires indivis d'un terrain (bien en immobilisation) acquis en 1985 au prix de 20 000.\$. Ce terrain vaut aujourd'hui 42 000.\$. En 1994, André et Bernard mettent fin à l'indivision en se partageant également le terrain. Dans cet exemple, le paragraphe 248 (20) L.I.R. stipule qu'il n'y a pas de disposition puisque chaque indivisaire détient maintenant en pleine propriété une moitié du terrain dont la valeur correspond à la valeur de sa part indivise dans le bien avant le partage.

EXEMPLE 2 : Reprenons maintenant le même exemple en modifiant cependant le partage. Suite au partage, André se retrouve seul propriétaire du terrain. Bernard, quant à lui, reçoit $21000 . \$$ soit la valeur de sa demie indivise. En vertu du paragraphe 248 (20) L.I.R., Bernard est réputé avoir disposé de sa part indivise dans le terrain ${ }^{19}$ pour une somme de $21000 . \$$. Bernard réalise donc un gain en capital de $11000 . \$$. Les trois quarts de ce gain seront ajoutés à son revenu de l'année. Les conséquences fiscales pour André sont les suivantes : André est réputé : a) avoir acquis la part indivise de Bernard à un montant équivalent à la juste valeur marchande de cette part immédiatement avant le transfert ${ }^{20}$ et b) ne pas avoir disposé de la part indivise qu'il possédait dans le terrain immédiatement avant le transfert ${ }^{21}$. Le partage n'occasionne aucun impact fiscal immédiat pour

18. Les paragraphes relatifs aux conséquences fiscales des partages ont été adoptés en 1991 et sont applicables aux partages effectués après le 13 juillet 1990 . Supra, note 9 .

19. Parce que Bernard ne détient pas au moins un droit de propriété correspondant à la valeur de sa part indivise avant le partage.

20. Parce que la proportion de son droit de propriété dans le bien détenu en indivision a augmenté suite au partage.

21. Parce que la proportion de son droit de propriété dans le bien n'a pas diminué suite au partage. 
André qui ajoute la valeur marchande de la part indivise appartenant à Bernard au prix de base rajusté de sa propre demie indivise, de sorte que le prix de base rajusté pour la totalité du terrain devient donc de $31000 . \$ 22$.

EXEMPLE 3 : $\quad$ André et Bernard sont propriétaires indivis des terrains 24 et 55 d'une valeur de 20000 .\$ chacun. Ils ont été acquis en 1987 au prix de 2000 . $\$$ chacun. Suite au partage, André reçoit le terrain 24 et Bernard le terrain 55. En vertu du paragraphe 248 (20) L.I.R., André est réputé avoir disposé à la juste valeur marchande de ses droits dans le terrain 55 et avoir acquis à la juste valeur marchande les droits de Bernard dans le terrain 24. En conséquence, il réalise un gain en capital de 9000 .\$ sur la disposition réputée de sa part du terrain 55 et le prix de base rajusté de la totalité du terrain 24 qu'il a conservé est de 11 000.\$. Les conséquences fiscales pour Bernard sont les mêmes relativement au terrain 24; il réalise un gain en capital de $9000 . \$$ sur la disposition réputée de sa part du terrain 24 et le prix de base rajusté du terrain 55 est fixé à $11000 . \$$.

Dans ce dernier exemple, chacun des indivisaires doit payer immédiatement les impôts rattachés à la plus-value du bien «partagé» qu'il ne possède plus, alors que dans l'exemple 1, aucun impact fiscal ne résulte du partage. Comme nous pouvons le constater, deux partages en nature de biens détenus en indivision n'engendrent pas nécessairement le même impact fiscal. Cette différence peut s'expliquer du fait que l'un des indivisaires reçoit en paiement un bien autre qu'une partie du bien détenu en indivision et qu'il ne s'agit donc pas dans les faits d'un partage en nature du seul bien détenu en indivision.

Les règles fiscales spécifiques aux partages diffèrent donc des règles civiles. En effet, même si, au plan civil, un partage était déclaratif de propriété, des conséquences fiscales pouvaient quand même en résulter selon l'issue du partage intervenu. Qu'en est-il depuis l'adoption du Code civil du Québec?

\section{C) Situation depuis le 1er janvier 1994}

L'alinéa 3 de l'article 1037 C.c.Q. stipule qu'un partage, autre que successoral, est attributif du droit de propriété. Cette nouvelle règle ne change en rien les conséquences fiscales étudiées ci-dessus. En effet, le paragraphe 248(20) L.I.R. s'applique à tous les partages qu'ils soient déclaratifs ou translatifs 
du droit de propriétée ${ }^{23}$. L'impact fiscal est toujours déterminé par le partage intervenu peu importe les règles civiles. Peut-on appliquer les mêmes présomptions aux autres législations fiscales?

\section{II.- $\quad$ Autres lois de nature fiscale}

\section{A) Loi concernant les droits sur les mutations immobilières ${ }^{24}$}

L'article 2 de la Loi concernant les droits sur les mutations immobilières stipule que :

«Toute municipalité doit percevoir un droit sur le transfert de tout immeuble situé sur son territoire, calculé en fonction de la contrepartie de ce transfert, selon les taux suivants :...»

Un partage d'un immeuble détenu en indivision est-il assujetti à cette loi? La réponse dépendra de l'interprétation du mot «transfert» contenu à l'article 2 mentionné ci-dessus ${ }^{25}$. Avant l'entrée en vigueur du Code civil du Québec, certains auteurs étaient d'avis que le partage d'un bien détenu en indivision ne constituait pas un «transfert» au sens de la Loi concernant les droits sur les mutations immobilières. Cette conclusion reposait sur le fait qu'un partage était déclaratif, et non translatif ${ }^{26}$, du droit de propriété. Conséquemment, ces auteurs soutenaient qu'un partage n'était pas assujetti à cette loi. Cette interprétation peut-elle être maintenue depuis le 1er janvier 1994 ?

Le Code civil du Québec stipule que le partage est maintenant «attributif » du droit de propriété. Le législateur n'utilise pas le terme «translatif» du droit de propriété ce qui aurait eu l'avantage de déterminer clairement qu'un partage effectué depuis l'entrée en vigueur du Code civil du Québec serait assujetti aux droits de mutation. Comment le mot «attributif» doit-il être interprété?

À notre avis, lorsqu'un bien détenu en indivision est partagé en nature entre les indivisaires et que leur part respective dans le bien après le partage correspond à la valeur de leur part indivise dans ce même bien, il n'y a pas de

23. Voir le paragraphe introductif de 248 (20) L.I.R.

24. Supra, note 11.

25. Le mot «transfert» est défini à l'article 1 de la loi : « «transfert» : le transfert du droit de propriété d'un immeuble ainsi que le contrat de louage d'un immeuble, pourvu que la période qui court à compter de la date du transfert jusqu'à celle de l'arrivée du terme du contrat de louage, y compris toute prolongation ou tout renouvellement y mentionné, excède 40 ans; le mot transfert ne comprend pas le transfert fait dans le seul but de garantir le paiement D.-C. LAMONTAGNE, supra, note 17. 
transfert de propriété. Dans les faits, les indivisaires ont «borné ou cristallisé» leur droit de propriété sur une portion précise du bien sans rien ajouter ou soustraire. Dans une telle situation, le terme «attributif» ne serait pas synonyme de «translatif» du droit de propriété. Il faut également conclure de cette interprétation que toute augmentation ou diminution de valeur entraînera un transfert au sens de la loi. En effet, dans ce dernier cas, les indivisaires ont modifié la «portion» de leur droit de propriété dans le bien suite au partage. L'avantage de cette interprétation au niveau fiscal est qu'un partage qui serait considéré comme un transfert en vertu de la Loi de l'impôt sur le revenu serait également un transfert selon la Loi concernant les droits sur les mutations immobilières; il y aura donc taxation dans les mêmes circonstances.

À l'inverse, si le mot «attributif» est interprété par les tribunaux comme translatif du droit de propriété dans tous les cas de partages ${ }^{27}$, une municipalité serait en droit d'exiger des droits de mutation et ce, peu importe l'issue du partage. Nul doute que ce résultat causera beaucoup de tracas aux praticiens. Illustrons cette problématique en reprenant les trois exemples de partage vus précédemment :

EXEMPLE 1 : $\quad$ André et Bernard sont copropriétaires indivis d'un terrain acquis en 1985 au prix de $20000 . \$$. Ce terrain vaut aujourd'hui 42 000.\$. En 1994, André et Bernard mettent fin à l'indivision en se partageant également le terrain. André devra payer des droits de mutation sur une contrepartie égale à $21000 . \$$ et Bernard devra en faire de même puisque dans les deux cas, il y a eu «transfert» d'une demie indivise au sens de la Loi concernant les droits sur les mutations immobilières bien qu'il n'y ait pas de disposition réputée au sens de la Loi de l'impôt sur le revenu ${ }^{28}$

EXEMPLE 2: $\quad$ Suite au partage, André se retrouve seul propriétaire du terrain. Bernard, quant à lui, reçoit la valeur de sa demie indivise, $21000 . \$$. André devra payer des droits de mutation sur une contrepartie égale à 21000 . (soit sur la valeur de son acquisition). Bernard, quant à lui, n'a pas de droits de mutation à payer puisqu'il n'acquiert aucun immeuble. Il doit cependant inclure le gain en capital réalisé suite au partage, alors qu'André ne réalise aucun gain en capital

27. Au soutien de cette interprétation, on pourrait prétendre que dès qu'il y a partage entre deux indivisaires, les deux parties disposent de leurs droits indivis et que ces droits deviennent un droit de propriété «entier» pour chacun des ex-indivisaires. De plus, le fait qu'un partage autre que successoral n'a plus d'effet rétroactif depuis le $1^{\text {er }}$ janvier 1994 est un autre argument en faveur de l'interprétation qu'un partage constitue un transfert. Supra, note 1, p. 606.

28. Ibid., p. 4 
immédiat puisqu'il n'y a pas eu disposition au sens de la Loi de l'impôt sur le revenu.

EXEMPLE 3 : $\quad$ André et Bernard sont propriétaires indivis des terrains 24 et 55 d'une valeur de 20000 .\$ chacun. Ils ont été acquis en 1987 au prix de 2000 . $\$$ chacun. Suite au partage, André reçoit le terrain 24 et Bernard le terrain 55. André doit payer des droits de mutation sur une contrepartie égale à 10000 . \$ pour l'acquisition de la moitié indivise du terrain 24. Bernard devra faire de même pour l'acquisition de la moitié indivise du terrain 55. Dans ce dernier exemple, l'impôt sur le revenu et les droits de mutation s'appliquent aux deux individus.

\section{B) Loi sur la taxe d'accise (partie IX - taxe sur les produits et services) ${ }^{29}$}

Tout «acquéreur d'une fourniture taxable effectuée au Canada doit payer à Sa Majesté du chef du Canada une taxe égale à $7 \%$ de la valeur de la contrepartie de la fourniture $»^{30}$. Un partage constitue-t-il une fourniture au sens de la loi? Le paragraphe 123 (1) définit le mot «fourniture» de la façon suivante :

«Sous réserve des articles 133 et 134, livraison de biens ou prestation de services, notamment par vente, transfert, troc, échange, louage, licence, donation ou aliénation.»

À la lumière de cette définition, il faut également s'interroger sur les effets du partage (y a t-il transfert ou non?). Cette question a été soulevée en 1991 lors d'une table ronde portant sur la T.P.S. et la T.V.Q. À cette époque, les représentants du ministère du Revenu du Canada étaient d'avis qu'un partage en droit québécois ne constituait pas une «fourniture de biens au sens de la loi en raison de son effet déclaratif ${ }^{31}$ et qu'en conséquence le ministère ne taxerait pas une telle transaction. Cette interprétation du ministère doit-elle être mise de côté depuis le $1^{\text {er }}$ janvier 1994? Comme nous pouvons le constater, la question relative à l'interprétation du mot «attributif» se pose encore une fois ${ }^{32}$. À notre avis, il doit être interprété selon les mêmes principes établis précédemment relativement à la Loi concernant les droits sur les mutations immobilières.

\footnotetext{
29. Supra, note 12 .

30. Par. 165 (1) L.T.A.

31. Table ronde sur la T.P.S. / T.V.Q., APFF, Congrès 91,1472, à la page 1497. Me Michel Turcot et Me Richard Chagnon sont également de cet avis. Voir Michel TURCOT et Richard CHAGNON, «L'impact des dispositions particulières de la T.P.S. et de la T.V.Q. sur la pratique notariale», R.D. - Fiscalité -Doctrine - Document 2 - p. 57 Voir TURCOT et CHAGNON, ibid., p. 7.
} 
Cependant, tant que le traitement fiscal d'un partage ne sera pas clairement établi, il faudrait, à titre de règle de prudence, considérer que depuis le $1^{\text {er }}$ janvier 1994, tout partage constitue un transfert au sens du paragraphe 123(1) de la Loi sur la taxe d'accise et qu'il est donc assujetti à la T.P.S.

L'étude de ces lois nous permet de conclure que tous les aspects fiscaux d'un partage doivent être envisagés avant de déterminer les parts de chacun des indivisaires ${ }^{33}$; ce qui inclut les incidences de toutes lois fiscales susceptibles de s'appliquer. Cependant, le partage n'est pas le seul élément de l'indivision qui vaut la peine d'être considéré. Certaines dispositions du système légal d'indivision soulèvent d'autres problèmes d'ordre fiscal et pratique. Examinons brièvement ces dispositions.

\section{III.- Certaines dispositions du régime légal d'indivision à éviter}

\section{A) Le droit de retrait de l'article 1022 C.c.Q.}

Ce droit de retrait permet à un indivisaire de racheter la part qu'une tierce personne étrangère à l'indivision aurait acquise à titre onéreux en lui remboursant le prix de la cession et les frais qu'elle a acquittés. Ce droit de retrait comporte, à notre avis, les désavantages suivants:

i) s'il s'agit d'un immeuble, les droits de mutation seront exigibles lors des deux transactions; soit la vente de la part indivise et le retrait;

ii) s'il s'agit d'une fourniture taxable au sens de la Loi sur la taxe d'accise, la T.P.S. serait exigible lors des deux cessions ${ }^{34}$;

iii) le dédoublement des honoraires professionnels et, en matière immobilière, le dédoublement des frais de publication;

Le droit de retrait soulève aussi d'autres questions importantes : quels sont les frais acquittés par la tierce partie qui seront remboursés par l'indivisaire se prévalant du droit de retrait? Doit-on inclure les honoraires professionnels, les frais de publication, les droits de mutation, la T.P.S. lorsqu'elle n'est pas remboursable à titre de crédit sur intrants etc.?

33. Nous avons choisi de restreindre l'étude de l'impact fiscal d'un partage aux deux législations ci-dessus étudiées puisque l'étude d'autres lois à caractère fiscal nous mènerait à des conclusions semblables. Par exemple, voir la Loi concernant les droits sur les transferts de terrains, L.R.Q., c. D-17, la Loi sur la taxe de vente du Québec, L.Q. 1991, c. 67.

34. Cet inconvénient pourrait cependant être éliminé dans les cas où le ou les cessionnaires pourrai(en)t réclamer un crédit de taxe sur intrants pour ces transactions, voir arts 169 et 170 L.T.A. 
Pour toutes ces raisons, il serait préférable d'inclure à toute convention d'indivision un droit de préemption ${ }^{35}$ et ainsi mettre de côté le système légal d'indivision sur ce point.

\section{B) La cessation de l'indivision (partage, art. 1033 C.c.Q.)}

L'article 1033 C.c.Q. stipule que l'indivisaire qui s'objecte au maintien de l'indivision peut être désintéressé par les autres indivisaires soit en nature, soit en numéraire. Comme nous l'avons vu précédemment, un partage en nature n'aura pas nécessairement le même impact fiscal qu'un partage en numéraire. Dans certains cas, un indivisaire pourrait être avantagé au détriment des autres. Afin d'éviter une telle situation ${ }^{36}$, il faudrait inclure à la convention d'indivision les modalités du partage à intervenir et déterminer immédiatement quel en serait l'impact fiscal. La convention mettrait donc de côté les dispositions des articles 1031 et 1033 C.c.Q. et aurait l'avantage d'accélérer le processus de partage.

\section{Conclusion}

L'ajout au Code civil du Québec d'un chapitre traitant spécifiquement de la copropriété par indivision a permis de combler un vide législatif. Bien que cette intervention législative ait été indispensable, nul ne peut ignorer l'impact des lois fiscales tant sur le régime légal de l'indivision que sur les copropriétés par indivision conventionnelles. Cet impact est d'autant plus important à considérer lorsque le traitement réservé aux partages diffère d'une loi fiscale à l'autre. En effet, comment justifier qu'un même partage ne soit pas imposable en vertu de la Loi de l'impôt sur le revenu mais qu'il le soit au sens de la Loi sur la taxe d'accise ou de la Loi concernant les droits sur les mutations immobilières? Cette étude permet de constater que bien qu'il soit impossible d'uniformiser complètement les législations de natures civile et fiscale, il serait important que le législateur harmonise à tout le moins les législations de nature fiscale.

35. L'article 1022 C.c.Q. prévoit expressément cette possibilité.

36. Étant donné l'impact fiscal potentiel vu ci-dessus. 
(1993) 24 R.D.U.S.

\section{8 (20) L.I.R.}

Partage de biens. Sous réserve des paragraphes (21) à (23) et pour l'application de la présente loi, dans le cas où un bien qui est la propriété conjointe de plusieurs personnes fait l'objet d'un partage à un moment donné, les règles suivantes s'appliquent malgré les effets rétroactifs ou déclaratoires d'un tel partage :

a) chacune de ces personnes qui avait un droit sur le bien immédiatement avant ce moment est réputée ne pas avoir disposé, à ce moment, de la fraction du droit, ne dépassant pas un, représentée par le rapport entre la juste valeur marchande du droit immédiatement après ce moment et sa juste valeur marchande immédiatement avant;

b) chacune de ces personnes qui a un droit sur le bien immédiatement après ce moment est réputée ne pas avoir acquis, à ce moment, la fraction du droit représentée par le rapport entre la juste valeur marchande du droit immédiatement avant ce moment et sa juste valeur marchande immédiatement après;

c) chacune de ces personnes qui avait un droit sur le bien immédiatement avant ce moment est réputée avoir eu, jusqu'à ce moment, la fraction du droit à laquelle l'alinéa $a$ ) ne s'applique pas et en avoir disposé à ce moment;

d) chacune de ces personnes qui a un droit sur le bien immédiatement après ce moment est réputée ne pas avoir eu, avant ce moment, la fraction du droit à laquelle l'alinéa $b$ ) ne s'applique pas et l'avoir acquis à ce moment;

e) les alinéas $a$ ) à $d$ ) ne s'appliquent pas s'il s'agit d'un droit sur un bien corporel fongible figurant à l'inventaire de la personne.

Pour l'application du présent paragraphe, la juste valeur marchande, à un moment donné, d'un droit sur le bien qui est un droit indivis est réputée égale au produit de la juste valeur marchande du bien à ce moment par le rapport entre ce droit et tous les droits indivis dans le bien. 